RESEARCH NOTE

\title{
A Modified Hybrid Algorithm of Set-Based Particle Swarm Optimization for Traveling Salesman Problem
}

\author{
Tao Liu and Michiharu Maeda \\ Fukuoka Institute of Technology, 3-30-1 Wajiro-higashi, Higashi-ku, Fukuoka 811-0295, Japan \\ E-mail: maeda@fit.ac.jp
}

\begin{abstract}
This paper is concerned with the discrete combinatorial optimization problem. A modified hybrid algorithm that combines the max-min ant system and set-based particle swarm optimization (MSPSO) is proposed to solve the traveling salesman problem (TSP). To avoid a local solution, the cooperative mechanism among individuals is applied in MSPSO. MSPSO is based on set-based particle swarm optimization (SPSO). Before the execution of SPSO, max-min ant system (MMAS) is executed to accelerate the convergence of the algorithm. The results of the numerical experiments show that the proposed approach is effective for solving the TSP.
\end{abstract}

Keywords: traveling salesman problem, set-based particle swarm optimization, max-min ant system

\section{Introduction}

Particle swarm optimization (PSO) is a population-based stochastic optimization technique developed by Kennedy and Eberhart in 1995, inspired by the social behavior of bird flocking and fish schooling [1]. PSO has been shown to perform well and is widely used to solve continuous problems. It has successfully solved a number of continuous optimization problems, and attempts have been made recently to extend it to discrete optimization problems.

Much research has been done on extending PSO to solve discrete combinatorial optimization problems, and several approaches have been developed. According to the research so far, these approaches for discrete particle swarm optimization (DPSO) can be classified into four types. The first is the discrete binary version, which is the first discrete version that was proposed by Kennedy and Eberhart [2]. The second is based on the swap operator outlined by Clerc and is applied to solve small TSPs [3]. In the third type, the position and velocity are defined as a fuzzy matrix. To obtain a feasible solution to the problem, the algorithms require a defuzzification method to decode the fuzzy matrix into a feasible solution [4]-[6]. The fourth incorporates some metaheuristics, or problemdependent local search techniques; the proposed algorithm is of this type [7]-[9]. The performances of the first three types are unsatisfactory. They are applicable only to the simple problems. For the TSP, if the number of cities is large, they are not applicable. The accuracy is poor and the computational cost is expensive. The fourth type is a hybrid DPSO approach. Metaheuristics are applied in it, so this type can be applied to complicated problems, the accuracy is good, and the computational cost is low. The performance of the fourth type is better than those of the first three types, but the structure of the fourth type is complicated, and hence, it is generally designed for specific problems.

Ant colony optimization (ACO) is a cooperative search algorithm inspired by the behavior of real ants. The max-min ant system (MMAS) is one kind of common extension of $\mathrm{ACO}$ and is thought to be the most accurate extension of ACO [10]. In the TSP, which is one of the most well-known combinatorial optimization problems, one must determine the shortest path that joins a given set of $\mathrm{N}$ points [11].

The modified hybrid algorithm proposed in this paper is based on set-based particle swarm optimization (SPSO) but also includes the additional execution of a max-min ant system (MMAS) to accelerate the convergence of the program. This proposed algorithm is abbreviated as MSPSO. To avoid a local solution, the cooperative mechanism among individuals is applied in SPSO. By the proposed approach, good results are obtained and the convergence is faster than in the tra- 
ditional approach, which is shown by the numerical experiments.

\section{Set-Based Particle Swarm Optimization}

This section outlines the novel, generic SPSO algorithm. The algorithm is described below. In SPSO, the position and velocity have been redefined [7]-[9].

Position: the position $(x, y)$ represents edge $(x, y)$ connecting cities $x$ and $y$.

Velocity: the velocity $(x, y) / X$ represents edge $(x, y)$ connecting cities $x$ and $y$ with the chosen probability $X$.

Multiplication Operator: Between a Coefficient and a Set with Possibilities (Coefficient $\times$ Velocity): Given a coefficient $\mathrm{c}(\mathrm{c} \geq 0)$ and a set with the chosen possibility $V=(x, y) / X$, their product is defined as $c V=c \times X$.

$$
c \times X= \begin{cases}1 & \text { if } c \times X>1 \\ c \times X & \text { otherwise }\end{cases}
$$

Subtraction Operator: Position A - Position B: A difference between two position vectors is defined as a set of edges that exist in A but not in B.

Addition Operator: Velocity + Velocity: Given two sets with possibilities $V_{1}=\left(x_{1}, y_{1}\right) / X_{1}$ and $V_{2}=\left(x_{2}, y_{2}\right) / X_{2}$, if $x_{1}=x_{2}$ and $y_{1}=y_{2}$, the possibility in $V_{1}+V_{2}$ is set to the larger one among $V_{1}$ and $V_{2}$.

On the basis of the above definitions, Eq. (1) is utilized for velocity updating.

$$
\begin{aligned}
& v_{i d}(t+1)= \omega \times v_{i d}+c_{1} \times r_{1} \times\left(p_{i d}-x_{i d}(t)\right) \\
&+c_{2} \times r_{2} \times\left(p_{g d}-x_{i d}(t)\right) \\
& i \in(1,2, \ldots, m), d \in(1,2, \ldots, n)
\end{aligned}
$$

where, $\omega$ is the inertia weight factor, $r_{1}$ and $r_{2}$ are random values in the range $[0,1], v_{i d}$ and $x_{i d}$ are the current velocity and position of the particle, respectively, $p_{i d}$ is the best solution this particle has reached, $p_{g d}$ is the current global best solution of all the particles, $c_{1}$ and $c_{2}$ are learning factors, $m$ is the swarm size, and $n$ is the number of particles in each swarm.

In the position-updating procedure, particle $i$ first obtains some desirable elements from $V_{i}$. In each iteration, a random number $\alpha \in(0,1)$ is generated for each particle to construct a remain set. For each element $(x, y) / X$ in $V_{i}$, if its corresponding possibility $X$ is equal or greater than $\alpha$, this element will be reserved into a remain set but not if $\alpha>\mathrm{X}$. The elements in the remain set will be chosen to construct the path. As only a symmetric TSP is discussed in this paper, $(x, y)$ is not different from $(y, x)$.

At the beginning of the algorithm, the starting city is initialized randomly. Choose edge that go through the city from the remain set according to the probability to construct a complete path. If the construction of a path is not finished and there is no available edge in the remain set, add the absent edge with the nearest principle.

\section{Max-Min Ant System}

ACO is a population-based approach that has been successfully applied in several fields. One of its main ideas is the indirect communication among the individuals of a colony of agents based on an analogy with trails of a chemical substance [10].

MMAS is one kind of common extension of ACO. MMAS differs from ACO [11]. In MMAS, to exploit the best solutions found during an iteration or during the run of the algorithm, after each iteration, only one single ant adds a pheromone. To avoid stagnation of the search, the range of possible pheromone trails for each solution component is limited to a certain interval $\left[\tau_{\max }, \tau_{\min }\right]$. Additionally, we deliberately initialize the pheromone trails to $\tau_{\max }$, thereby achieving greater exploration of solutions at the start of the algorithm.

\section{Cooperative Mechanism for Set-Based Particle Swarm Optimization}

In the conventional SPSO, every particle updated its velocity and position according to its best solution and the global best solution. Because of the influence of the global best solution, every particle will quickly converge about the global best solution. If the global best solution is a local one, the colony will fall into the local best solution, and will not emerge from it easily. Studies show that particles converge rapidly at the initial evolutional stages, whereas as the iteration progresses, most particles of the swarm will gradually become the same, and the global optimum is difficult to find. The reason for this phenomenon is that each particle considers information of only itself and the best particles of the swarm during the search process. The particle searches only the global optimum and its neighborhood thoroughly, and does not fully exploit other areas. Additionally, in the basic PSO algorithm, the initialization greatly affects the final result. It is difficult to obtain the global optimal solution if each particle is far from it after initialization $[12,13]$.

In the modified algorithm, a cooperative mechanism among individuals has been applied. Particles not only adjust their own flying speed according to their own information and that of the best individual of the swarm, but also learn from other individuals according to a certain probability. At the initial stage of evolution, the particle learns from individuals with high probability, whereas at the end of the iteration, it learns from the best individuals of the swarm with 
high probability. Chances are high that particles will find the global optimum.

The selection policy is given below.

$\gamma$ is a randomly generated number between 0 and 1. $R_{t}$ is calculated according to Eq. (2). If $\gamma>R_{t}$, choose a particle randomly other than itself and the best one and use its position. Record that particle as $P_{r n d}$ in place of $P_{g d}$ in the equation and update the particle's velocity so that the Eq. (1) becomes Eq. (3); else, update the velocity by the traditional approach [8].

$$
\begin{gathered}
R_{t}=\frac{t}{T_{\text {Max }}} \\
v_{i d}(t+1)=\omega \times v_{i d}+c_{1} \times r_{1} \times\left(p_{i d}-x_{i d}(t)\right) \\
+c_{2} \times r_{2} \times\left(p_{\text {rnd }}-x_{i d}(t)\right)
\end{gathered}
$$

where, $t$ is the current generation and $T_{\text {Max }}$ is the maximum number of iterations of the algorithm.

\section{Max-Min Ant System and Set-Based Particle Swarm Optimization with Cooperative Mechanism}

The proposed MSPSO is based on SPSO. The convergence speed of MMAS is faster than that of SPSO, so MMAS is executed before SPSO. Both MMAS and SPSO have the possibility of lapsing into a local solution. To avoid the local solution, the cooperative mechanism among individuals is applied in SPSO. Firstly, MMAS is executed and the global solution is obtained. Then, particles are initialized, and then in accordance with Eqs. (1) and (3), the velocity is updated using the global solution obtained by MMAS. SPSO is executed until the maximum number of iterations. The procedure of MSPSO is shown in Fig. 1 .

In previous SPSO schemes, particles only update the velocity and position according to the local solution and the global solution. After the cooperative mechanism is applied into the proposed algorithm, the particles update the velocity and position not only according to the global solution but also according to randomly chosen particle other than themselves and the global solution. The search range becomes wide and the diversity of the solution is maintained.

\section{Numerical Experiment}

The numerical experimental results are presented in this section. The accuracy is compared among ACO, MMAS, SPSO, SPSO with the cooperative mechanism (SPSOCM), and MSPSO with the cooperative mechanism for the cases of eil76, Berlin52 and KroA100 from the traveling salesman problem library (TSPLIB) [14].

The swarm number can also influence the performance the PSO algorithm. In general, a small swarm

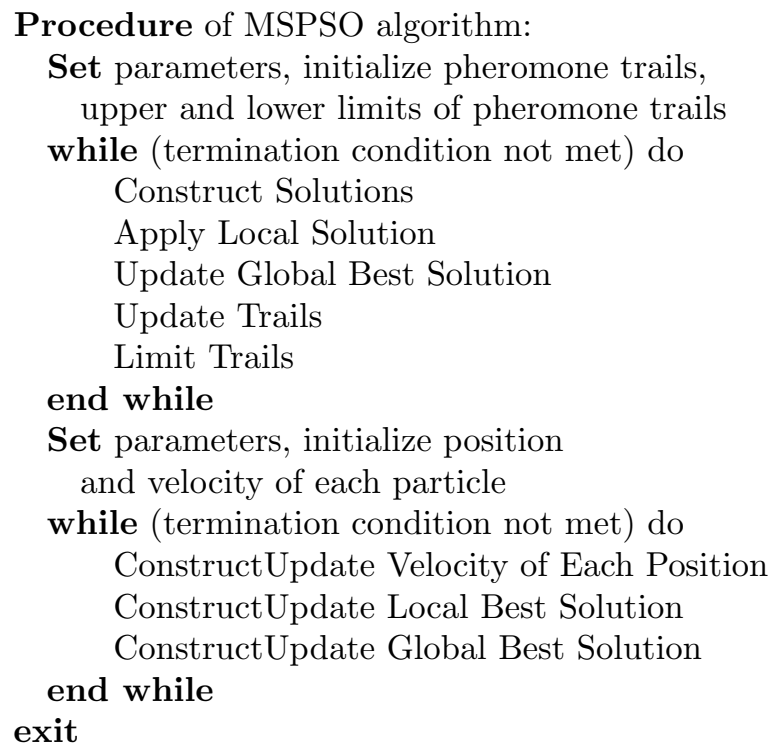

Fig. 1 Scheme of MSPSO.

can accelerate the search process, but the diversity of the population is reduced. On the other hand, a large swarm results in higher diversity of the population, but the computational effort in each iteration significantly increases, and thus the search process becomes slow. For the TSP, it can be seen that when $\mathrm{M}=20$, 30 , and 40 , the best results are achieved according to previous studies. In the experiments in this study, the intermediate value $\mathrm{M}=30$ is applied.

For $\omega$, if $\omega$ is small, there will be fewer elements in the remain set for the particle to learn from. Oppositely, if $\omega=1$, the elements in the previous $\mathrm{V}$ will not be forgotten, and thus there will be too many elements in the remain set for the particle to learn from. According to the previous studies, a value of $\omega$ that decreases from 0.9 to 0.4 linearly is applied [12]. The equation for $\omega$ is described below.

$$
\omega=0.9-\frac{t}{T_{\text {Max }}} \times 0.5
$$

where, $t$ is the current generation, and $T_{\mathrm{Max}}$ is the maximum number of iterations of the algorithm.

The acceleration coefficient $c$ indicates the importance of the newly learnt elements. A larger c will have a better chance of remaining in the remain set with possibility $c \times r$. According to the previous studies, if $c$ is small, particles learn from only a few elements in the remain set. If $c$ is greater than 1.0, the diversity and convergence of the algorithm can be balanced, and the algorithms perform well. Hence $c=2.9$ is adopted in the experiments.

Table 1 shows, for eil76, the results of the comparison among ACO, MMAS, SPSO, SPSOCM, and MSPSO with the cooperative mechanism. 
Table 1 Results for eil76 obtained by ACO, MMAS, SPSO, SPSOCM and MSPSO

\begin{tabular}{|c|c|c|c|}
\hline Algorithm & Worst & Best & Mean \\
\hline ACO & 572.495 & 559.198 & 569.7317 \\
\hline MMAS & 570.62 & 559.637 & 565.5689 \\
\hline SPSO & 583.292 & 552.499 & 562.9482 \\
\hline SPSOCM & 581.543 & 552.841 & 563.3476 \\
\hline MSPSO & 573.399 & 549.17 & 563.4259 \\
\hline
\end{tabular}

The optimal value in the case of eil76 is 538 . The results in Table 1 are averages of 1000 runs. The maximum number of iterations in each run is 3000 . As shown in Table 1, the best mean value is obtained by SPSO, but the minimum of the best values is obtained by the proposed approach, that is, MSPSO with the cooperative mechanism.

Table 2 shows, for Berlin52, the results of the comparison among ACO, MMAS, SPSO, SPSOCM, and MSPSO with the cooperative mechanism.

Table 2 Results for Berlin52 obtained by ACO, MMAS, SPSO, SPSOCM and MSPSO

\begin{tabular}{|c|c|c|c|}
\hline Algorithm & Worst & Best & Mean \\
\hline ACO & 7782.17 & 7548.99 & 7686.009 \\
\hline MMAS & 7775.87 & 7548.99 & 7682.285 \\
\hline SPSO & 7887.04 & 7544.37 & 7555.654 \\
\hline SPSOCM & 7887.04 & 7544.37 & 7558.628 \\
\hline MSPSO & 7719.91 & 7544.37 & 7550.931 \\
\hline
\end{tabular}

The optimal value for Berlin52 is 7542 . The results in Table 2 are averages of 1000 runs. The maximum number of iterations in each run is 2000. As shown in Table 2, the minimum best values obtained by SPSO, SPSOCM, and MSPSO are the same, but the best mean value is obtained by the the proposed approach, MSPSO with the cooperative mechanism.

Table 3 shows, for KroA100, the results of the comparison among ACO, MMAS, SPSO, SPSOCM, and MSPSO with the cooperative mechanism.

Table 3 Results for KroA100 obtained by ACO, MMAS, SPSO, SPSOCM and MSPSO

\begin{tabular}{|c|c|c|c|}
\hline Algorithm & Worst & Best & Mean \\
\hline ACO & 23238.6 & 22319 & 22693.79 \\
\hline MMAS & 23360.7 & 22288.5 & 22680.95 \\
\hline SPSO & 22613 & 21406.8 & 21887.88 \\
\hline SPSOCM & 22591.1 & 21342.8 & 21734.94 \\
\hline MSPSO & 22475.8 & 21321.8 & 21721.4 \\
\hline
\end{tabular}

The optimal value for KroA100 is 21282 . The results in Table 3 are averages of 1000 runs. The maximum number of iterations in each run is 5000. In Table 3 , the minimum best value and the minimum mean value are both obtained by the proposed approach, MSPSO with the cooperative mechanism.

\section{Conclusion}

In this paper, the max-min ant system combined with set-based particle swarm optimization (MSPSO), in which the cooperative mechanism among individuals is applied, is proposed. Through numerical experiments for large-size TSPs of eil76, Berlin52, and KroA100, the performance of the proposed MSPSO with the cooperative mechanism was found to be better than those of ant colony optimization (ACO), the max-min ant system (MMAS), set-based particle swarm optimization (SPSO), and SPSO with the cooperative mechanism (SPSOCM). At the start of the proposed approach, MMAS is executed, so the convergence of the proposed approach is faster than SPSO and SPSOCM. Then, SPSO with the cooperative mechanism is executed to maintain the solution diversity. The difference in the results for KroA100 between the proposed approach and the traditional approaches is greater than in the results for eil76 and Berlin52. Therefore, the proposed approach is more effective for KroA100 than for eil76 and Berlin52. The cooperative mechanism among individuals can prevent the algorithm from falling into a local solution, so the accuracy of the proposed approach is better than those of ACO, MMAS, and SPSO. The parameter setting can also influence the algorithm. The results of the numerical experiments have shown that the proposed approach is promising.

\section{References}

[1] J. Kennedy and R. C. Eberhart: Particle swarm optimization, Proc. IEEE Int. Conf. Neural Netw., pp. 1942-1948, 1995.

[2] J. Kennedy and R. C. Eberhart: A discrete binary version of the particle swarm algorithm, Proc. IEEE Int. Conf. Syst. Man Cybern., pp. 4104-4109, 1997.

[3] M. Clerc: Discrete Particle Swarm Optimization, in New Optimization Techniques in Engineering, New York: Springer-Verlag, 2004.

[4] W. Pang, K.-P. Wang, C.-G. Zhou and L.-J. Dong: Fuzzy discrete particle swarm optimization for solving traveling salesman problem, Proc. 4th Int. Conf. Comput. Information Technol. (CIT), pp.796-800, 2004.

[5] B. Shen, M. Yao and W. Yi: Heuristic information based improved fuzzy discrete PSO method for solving TSP, Proc. 9th Pacific Rim Int. Conf. Artif. Intell. (PRICAI), pp. 856863, 2006.

[6] C.-J Liao, C.-T. Tseng and P. Luarn: A discrete version of particle swarm optimization flowshop scheduling problem, Comput. Operations Res., Vol. 34, No. 10, pp. 3099-3111, Oct. 2007. 
[7] Y.-X. Jin, H.-Z. Cheng, J.-Y. Yan and L. Zhang: New discrete method for particle swarm optimization and its application in transmission network expansion planning, Electr. Power Syst. Res., Vol. 77, Nos. 3-4, pp. 227-233, Mar. 2007.

[8] X. H. Shi, Y. C. Liang, H. P. Lee, C. Lu and Q. X. Wang: Particle swarm optimization-based algorithms for TSP and generalized TSP, Inform. Process. Lett., Vol. 103, No. 5, pp. 169-176, Aug. 2007.

[9] W.-N. Chen, J. Zhang, H.S.H. Chung, W.-L. Zhong, W.-G. Wu and Y.-H. Shi: A novel set-based particle swarm optimization method for discrete optimization problems, IEEE Trans. Evol. Comput., Vol.14, No.2, pp.278-299, Apr. 2010.

[10] M. Dorigo and T. Stutzle: Ant Colony Optimization, MIT Press, 2004.

[11] T. Stutzle and H. Hoos: MAX-MIN ant system, Future Generation Computer System, Vol.16, No.8, pp. 889-914, Jun. 2000.

[12] C. Wang, J. Zhang, J. Yang, C. Hu and J. Liu: A modified particle swarm optimization algorithm and its application for solving traveling salesman problem, IEEE Proc. Int. Conf. Neural Networks and Brain, Vol.2, pp.689-694, 2005.

[13] A. Rantnaweera, S. K. Halgamuge and H. C. Watson: Selforganizing hierarchical particle swarm optimizer with timevarying acceleration coefficients, IEEE Trans. Evol. Comput., Vol. 8, No. 3, pp. 240-255, Jun. 2004.

[14] G. Reinelt: TSPLIB: a traveling salesman problem library, Orbit Reconst. Simulation Anal. J. Comput., Vol. 3, No. 4, pp. 376-384, Jan. 1991.

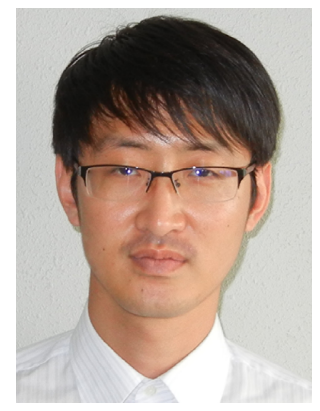

Tao Liu received his B.A. in English Language from Liaoning Institute of Technology, China, and his M.E. in computer science and engineering from Fukuoka Institute of Technology, Japan, in 2006 and 2012, respectively. Since 2012, he has been a doctorate student in the Graduate School of Intelligent Information System Engineering, Fukuoka Institute of Technology. His current research intertion and swarm intelligence. ests include evolutionary computa-

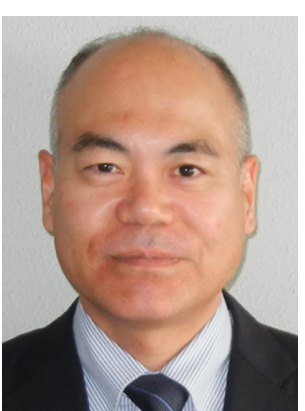

Michiharu Maeda received his B.S. and M.S. degrees in theoretical physics in 1990 and 1992, respectively, and his Ph.D. degree in information and computer science in 1997, from Kagoshima University, Japan. He is currently a Professor in the Department of Computer Science \& Engineering at Fukuoka Institute of Technology. His research interests include computational intelligence, mathematical and physical computation,

and signal processing.

(Received May 15; revised September 4, 2013) 